\title{
Pre-pregnancy body mass index and adverse maternal and perinatal outcomes in twin pregnancies: a population based retrospective cohort study
}

\author{
Jeffrey Bone ${ }^{1}$, K S Joseph ${ }^{2}$, Laura Magee ${ }^{3}$, Li Qing Wang ${ }^{1}$, Chantal Mayer ${ }^{4}$, and Sarka \\ Lisonkova $^{1}$ \\ ${ }^{1}$ The University of British Columbia \\ ${ }^{2}$ The University of British Columbia School of Population and Public Health \\ ${ }^{3}$ St. Georges, University of London \\ ${ }^{4}$ University of British Columbia
}

February 6, 2022

\begin{abstract}
Objectives To assess associations between pre-pregnancy BMI and severe maternal morbidity (SMM), perinatal death and neonatal morbidity in twin pregnancies. Design Retrospective cohort study. Setting British Columbia, Canada (2000-2017). Population All twin births at [?]20 weeks' gestation. Methods We examined associations between pre-pregnancy BMI, categorized as underweight (BMI $<18.5 \mathrm{~m} / \mathrm{kg} 2)$, normal BMI $(18.5 \mathrm{~m} / \mathrm{kg} 2$ ? BMI $<25 \mathrm{~m} / \mathrm{kg} 2)$, overweight $(25 \mathrm{~m} / \mathrm{kg} 2$ ? BMI $<30 \mathrm{~m} / \mathrm{kg} 2)$ and obese (BMI [?]30m/ kg2) and adverse maternal and perinatal outcomes, using targeted maximum-likelihood estimation, adjusted for demographics and obstetric history. Outcomes SMM, perinatal death, severe perinatal morbidity. Results Overall, 7770 (368 underweight, 1704 overweight and 1016 obese) women with twin pregnancy were included. The rates of SMM (per 10,000 pregnancies) were: 271.1, 320.4, 270.0 and 225.9 in underweight, normal BMI, overweight and obese women, respectively. Underweight women had higher rates of the composite perinatal adverse outcome (adjusted rate ratio) $[\mathrm{aRR}]=1.79,95 \% \mathrm{CI}=$ 1.32- 2.43), largely driven by increased rates of severe respiratory distress syndrome, and neonatal death $(\mathrm{aRR}=2.81,95 \% \mathrm{CI}$ $=1.64-4.83)$. There was no evidence of elevated risk for perinatal outcomes among overweight and obese women. Conclusions In this population-based study cohort study, we found no evidence of an increased risk of adverse maternal and perinatal outcomes in overweight and obese women with twins; however, infants of underweight women are at higher risk of neonatal death and morbidity. These findings have implications for pregnancy care of women with twin pregnancies, especially those who are underweight. Funding SickKids Foundation (SKF-154852) Keywords: pre-pregnancy obesity, maternal morbidity, perinatal death
\end{abstract}

Title: Pre-pregnancy body mass index and adverse maternal and perinatal outcomes in twin pregnancies: a population based retrospective cohort study

\section{Authors :}

Mr. Jeffrey N Bone MSc, KS Joseph ${ }^{2}$ MD, PhD, Laura A Magee MD, Ms. Li Qing Want BSc, Chantal Mayer MD, Sarka Lisonkova,2 MD, PhD

\section{Affiliations:}

1. Department of Obstetrics and Gynaecology, University of British Columbia and the Children's and Women's Hospital and Health Centre of British Columbia, Vancouver, BC, Canada.

2. School of Population and Public Health, University of British Columbia, Vancouver, BC, Canada. 
3. Department of Women and Children's Health, School of Life Course Sciences, Faculty of Life Sciences and Medicine, King's College London, London, UK

\section{Corresponding author:}

Jeffrey N. Bone, MSc,

Department of Obstetrics and Gynaecology

University of British Columbia

Children's and Women's Hospital and Health Centre

Room CSB V3-340, 950 West $28^{\text {th }}$ Avenue,

Vancouver, BC, V6A 3V4, Canada

E-mail: jeffrey.bone@cw.bc.ca

Short title: Pre-pregnancy BMI and adverse outcomes in twins

\section{ABSTRACT}

\section{Objectives}

To assess associations between pre-pregnancy BMI and severe maternal morbidity (SMM), perinatal death and neonatal morbidity in twin pregnancies.

\section{Design}

Retrospective cohort study.

\section{Setting}

British Columbia, Canada (2000-2017).

\section{Population}

All twin births at [?]20 weeks' gestation.

\section{Methods}

We examined associations between pre-pregnancy BMI, categorized as underweight (BMI $<18.5 \mathrm{~m} / \mathrm{kg}^{2}$ ), normal BMI $\left(18.5 \mathrm{~m} / \mathrm{kg}^{2}[?]\right.$ BMI $\left.<25 \mathrm{~m} / \mathrm{kg}^{2}\right)$, overweight $\left(25 \mathrm{~m} / \mathrm{kg}^{2}[?] \quad\right.$ BMI $\left.<30 \mathrm{~m} / \mathrm{kg}^{2}\right)$ and obese (BMI $\left.[?] 30 \mathrm{~m} / \mathrm{kg}^{2}\right)$ and adverse maternal and perinatal outcomes, using targeted maximum-likelihood estimation, adjusted for demographics and obstetric history.

\section{Outcomes}

SMM, perinatal death, severe perinatal morbidity.

\section{Results}

Overall, 7770 (368 underweight, 1704 overweight and 1016 obese) women with twin pregnancy were included. The rates of SMM (per 10,000 pregnancies) were: 271.1, 320.4, 270.0 and 225.9 in underweight, normal BMI, overweight and obese women, respectively. Underweight women had higher rates of the composite perinatal adverse outcome (adjusted rate ratio) $[\mathrm{aRR}]=1.79,95 \% \mathrm{CI}=1.32-2.43$ ), largely driven by increased rates of severe respiratory distress syndrome, and neonatal death $(\mathrm{aRR}=2.81,95 \% \mathrm{CI}=1.64-4.83)$. There was no evidence of elevated risk for perinatal outcomes among overweight and obese women.

\section{Conclusions}

In this population-based study cohort study, we found no evidence of an increased risk of adverse maternal and perinatal outcomes in overweight and obese women with twins; however, infants of underweight women 
are at higher risk of neonatal death and morbidity. These findings have implications for pregnancy care of women with twin pregnancies, especially those who are underweight.

\section{Funding}

SickKids Foundation (SKF-154852)

Keywords: pre-pregnancy obesity, maternal morbidity, perinatal death

\section{Tweetable abstract:}

In twin pregnancies, underweight, but not overweight or obese women have higher risk of worse outcomes compared with women of normal weight.

\section{INTRODUCTION}

Obesity is an important risk factor in pregnancy and its prevalence among pregnant women has been steadily increasing in recent decades. ${ }^{1}$ For example, in the United States, recent studies show that pre-pregnancy obesity is prevalent in $29 \%$ of women, up from $26 \%$ in $2016^{2}$. Similar trends have been observed in other high-income settings, as well as in developing countries. ${ }^{1,3,4}$ High pre-pregnancy body-mass-index (BMI) is associated with an increased risk of both poor maternal and perinatal outcomes. In singleton pregnancies, depending on the level of obesity, obese women (vs. those of normal BMI) are at 20-40\% increased risk of severe maternal morbidity $(\mathrm{SMM})^{5}$ and at $50 \%$ increased risk of stillbirth and neonatal death ${ }^{67}$

Multiple pregnancy is also a recognized risk factor for adverse pregnancy outcomes, compared with singleton pregnancy. Women with twin (vs. singleton) pregnancies experience up to four-fold higher rates of maternal complications, stillbirth rates that are five-fold (in dichorionic twins) to thirteen-fold higher (monochorionic twins), ${ }^{8,9}$ and similarly elevated rates of neonatal death and serious neonatal morbidity. ${ }^{8,10}$

Though there is ample evidence for higher pregnancy risks associated with obesity in singleton pregnancies, these pre-pregnancy BMI-specific risks are understudied in twin pregnancies. Previous studies in twin pregnancies have demonstrated an enhanced risk of obesity with regard to pre-eclampsia, gestational diabetes, and preterm birth. ${ }^{8,11,12}$ Relevant studies examining specific SMM conditions are scarce, possibly due to the need for large sample size to assess rare outcomes. Extrapolating from the known effects of BMI on adverse pregnancy outcomes in singletons, the highest rates of perinatal mortality would be expected in obese women with twins. However, some prior evidence suggests that the effect of BMI on adverse perinatal outcomes is modified in twins, and that the association between pre-pregnancy BMI and adverse perinatal outcomes including stillbirth, neonatal death and serious neonatal morbidity in twin pregnancies may be the inverse of what might be expected. ${ }^{11}$

The objective of this study was to examine the association between pre-pregnancy BMI and SMM, stillbirth, neonatal death, and severe neonatal morbidity in women with twin pregnancies and their infants.

\section{MATERIALS AND METHODS}

Data source and study population

This was a retrospective cohort study of all twin births at [?] 20 weeks' gestation in British Columbia, Canada, between 2000 and 2017. Data were obtained from the British Columbia Perinatal Database Registry (BCPDR), which contains individual level data on $>99 \%$ of births in British Columbia since 2000, including home births. ${ }^{13}$ Validation studies of BCPDR data against medical charts showed high accuracy of collected data. $^{14}$

The BCPDR includes information about maternal demographics, pre-pregnancy morbidity, BMI, and pregnancy-related data. The database also includes details about delivery and newborn hospitalizations with up to 25 diagnoses related to each hospitalization. The diagnoses are coded using International Classification of Diseases - Canadian modification version 10, ICD-10-CA - and up to 20 procedure codes (coded using the Canadian Classification of Interventions, CCI) for both the mother and newborn. In addition, the 
database contains information about hospital readmissions for mothers and babies for up to 42 and 28 days, respectively.

Our primary exposure of interest was pre-pregnancy BMI (based on self-reported height and pre-pregnancy weight), categorized in our primary analyses into standard groups, namely, underweight $\left(\mathrm{BMI}<18.5 \mathrm{~kg} / \mathrm{m}^{2}\right)$, normal BMI $\left(18.5 \mathrm{~kg} / \mathrm{m}^{2}\right.$ [?] BMI $\left.<25 \mathrm{~kg} / \mathrm{m}^{2}\right)$, overweight $\left(25 / \mathrm{kg} / \mathrm{m}^{2}\right.$ [?] BMI $\left.<30 \mathrm{~kg} / \mathrm{m}^{2}\right)$ and obese $(\mathrm{BMI}$ [?] $\left.30 \mathrm{~kg} / \mathrm{m}^{2}\right)$. Pregnancies at $<20$ weeks' gestation and those with missing information about pre-pregnancy BMI were excluded.

Outcome measures and potential confounders

We examined associations between pre-pregnancy BMI and both SMM and adverse perinatal outcomes. SMM was defined as a composite outcome that included potentially life-threatening conditions, either leading to serious sequelae, requiring intensive care unit admission, or death during the hospitalization for childbirth. A list of such conditions was previously developed by the Canadian Perinatal Surveillance System, ${ }^{15}$ and included: acute cardiac, hepatic, or renal failure, need for assisted ventilation, cerebrovascular morbidity, embolism, shock or disseminated intravascular coagulation (DIC), hysterectomy, surgical complications, severe pre-eclampsia (or eclampsia), maternal sepsis, severe hemorrhage or uterine rupture, and maternal death. The adverse composite perinatal outcome included stillbirth, neonatal death, perinatal death (stillbirth or neonatal death), and severe neonatal morbidity including intraventricular hemorrhage (grade 3,4), periventricular leukomalacia, necrotizing enterocolitis, sepsis, and severe birth trauma. (Tables S1 and S2 show the ICD-10 codes and details for SMM and severe neonatal morbidity, respectively).

We adjusted for variables identified previously as potential confounders in singleton pregnancies ${ }^{16,17}$ including maternal age, parity, self-reported smoking, alcohol or drug use during pregnancy, chronic hypertension, prepregnancy diabetes mellitus, other chronic pre-pregnancy conditions (e.g., cardiac, hepatic, renal conditions; Table S3) and prior stillbirth or neonatal death. For perinatal outcomes, additional confounders included congenital anomalies and infant sex.

Statistical analyses

Composite maternal outcomes were calculated per 10,000 pregnancies, fetal outcomes per 10,000 total births, and neonatal outcomes per 10,000 live births. For each BMI group, we computed adjusted risk ratios and risk differences (compared with normal BMI), using Targeted Maximum Likelihood Estimation (TMLE). ${ }^{18}$ TMLE has the advantage over traditional regression models in that it combines a propensity score model for the exposure, and another model for the outcome, thereby offering two opportunities to adjust for possible confounding. For both exposure and outcome models, we used an ensemble of traditional regression models and machine learning algorithms (i.e., 'stacking') to accommodate possible interactions and nonlinearities that common regression models alone may not include. These methods included: mean value regression, logistic regression (both main effects and all interactions), adaptive spline regression, generalized additive models, and tree-based models. For perinatal outcomes, we adjusted the standard errors for clustering within pregnancies, using previously published methods. ${ }^{19}$

We conducted several sensitivity analyses. First, we examined the dose-response relationship between prepregnancy BMI and adverse birth outcomes by analysing BMI as a continuous variable, using a restricted cubic spline with five knots. Results are presented as risk curves for the main adverse outcomes across the BMI spectrum. TMLE is not available for continuous exposures and therefore we used generalized estimating equations with exchangeable correlation structures for these models. Second, due to a relatively high rate of missing BMI, we conducted an analysis of our primary outcomes incorporating missingness into the TMLE routines described above. This process assumed that the data were 'Missing at Random' (MAR), implying that the missingness in BMI could be explained by the measured confounders. Third, we conducted a standard regression analysis using Poisson Generalized Estimating Equations (GEE) to accommodate for twin pregnancies, adjusting for the same confounders as in the TMLE. ${ }^{20}$ Finally, all analyses were repeated after restricting the study population to opposite-sex twins, in order to evaluate potential confounding by chorionicity. $^{21}$ 
All analyses were conducted using $\mathrm{R}$ statistical software and TMLE models were fit with the drtmle package. ${ }^{22,23}$

\section{RESULTS}

Of 724,920 births in British Columbia between 2000 and 2017, there were 22,066 twin births (3.0\%) from 11,033 pregnancies. Among women with twins, 3263 (29.6\%) had missing pre-pregnancy BMI. After this exclusion, the study population consisted of 7,770 women with twin pregnancy. Of these, 1016 women (13.1\%) were obese, 1704 (21.9\%) were overweight, $4682(60.3 \%)$ had normal BMI, and 368 (4.7\%) were underweight.

On average, women with twin pregnancies were 31 to 32 years of age (Table 1). Overweight and obese women were more likely to have prior hypertension, diabetes, caesarean delivery, stillbirth, or neonatal death. They were also more likely to be parous, smoke or use substances during pregnancy. Conception following the use of assisted reproductive technologies (ART) was similar across BMI categories. Pregnancy complications such as suspected intrauterine growth restriction (IUGR, based on ultrasound examination), anemia, and preterm birth at $<32$ weeks were more common among underweight women and complication rates were lower with increasing BMI. In contrast, other pregnancy complications such as gestational hypertension, gestational diabetes, and caesarean delivery were more common with in the higher BMI categories. Largefor-gestational age infants were more frequent among obese women.

SMM occurred in 222 pregnancies, at a rate of 285.7 per 10,000 twin pregnancies. SMM rates were highest among women with normal BMI (320.4 per 10,000 pregnancies; Table 2). The most common SMM was severe pre-eclampsia and/or eclampsia, which made up over a third of the composite SMM outcome. Other relatively frequent morbidities included severe hemorrhage (requiring transfusion), surgical complications, sepsis, and peripartum hysterectomy (Table 2).

The perinatal composite outcome occurred in 877 fetuses/infants (5.6\%), most commonly among underweight women (Table 2). Serious neonatal morbidity accounted for more than half of the composite outcome, largely due to bronchopulmonary dysplasia, severe respiratory distress syndrome (with mechanical ventilation for $>3$ days) and neonatal sepsis. Stillbirth rates were similar across BMI categories.

Adjusted analyses showed no clear association between pre-pregnancy BMI category and SMM (Table 3). However, the adjusted rate ratio (vs. normal BMI) was $<1.0$ for overweight (aRR 0.86, 95\% CI $=0.62$ to 1.19 ) and obese women ( $\mathrm{aRR} 0.77,95 \% \mathrm{CI}=0.50$ to 1.18$)$, and $>1.0$ for underweight women $(\mathrm{aRR}=$ $1.62,95 \% 0.83$ to 3.16 ), although confidence intervals were wide. Rate ratios comparing overweight and obese women versus women of normal BMI had confidence intervals that were centered narrowly around 1.0, indicating weak or no associations. Underweight women had elevated rates of the composite adverse perinatal outcome (aRR 1.79, 95\% CI $=1.32$ to 2.43) and all components. Absolute risk differences showed similar patterns of association (Table 3 ).

Dose-response curves between BMI as a continuous variable $\left(\mathrm{kg} / \mathrm{m}^{2}\right)$ and adverse pregnancy outcomes were largely flat between BMIs of $20 \mathrm{~kg} / \mathrm{m}^{2}$ and $40 \mathrm{~kg} / \mathrm{m}^{2}$. In underweight women, the risk of neonatal death and serious neonatal morbidity was similar to the higher risks noted in the categorized analyses above. There was a suggestion that neonatal morbidity was higher among women with BMI $>40 \mathrm{~kg} / \mathrm{m}^{2}$ but associations at very high of BMIs had very wide confidence intervals due to small numbers of women (Figure 1).

Demographic risk factors were similar between those with and without missing BMI (Figure S1). Sensitivity analyses including imputation for missing data and use of regression models (rather than TMLE) produced similar results (Tables S4 and S5). Additionally, when restricting our analyses to opposite sex twins, we found that adverse outcome rates were lower for all perinatal outcomes, while results for SMM were similar (Table S6). In the subgroup of opposite sex twins, adjusted associations were slightly attenuated in contrasts of obese women vs women with normal BMI, while results for underweight and overweight women were similar. Analyses of opposite sex twins yielded similar aRRs which had wider confidence intervals due to reduced study size (Table S7). 


\section{DISCUSSION}

\section{Principal findings}

This study involving a population-based cohort of twin pregnancies showed that the rates of SMM, perinatal death and neonatal morbidity were similar or lower in women who were overweight or obese prior to pregnancy and women who had a normal BMI. The narrow confidence intervals around the adjusted rate ratios indicated that large differences in maternal and fetal/infant risks associated with high maternal BMI are unlikely. This his finding was consistent with dose-response curves from the continuous BMI analysis, including at BMIs above $35 \mathrm{~kg} / \mathrm{m}^{2}$. In contrast, rates of SMM, perinatal death and neonatal morbidity were similar or higher in underweight women (compared with women of normal BMI), with underweight women having significantly higher rates of composite neonatal death or severe neonatal morbidity (79\% higher risk, 95\% CI 32-143) and severe neonatal morbidity ( $74 \%$ higher risk, $95 \%$ CI 17-159). Underweight women had especially high rates of neonatal death (181\% higher risk, 95\% CI 64-383).

Results in the context of what is known

Prior literature assessing pre-pregnancy BMI-specific rates of severe adverse maternal and perinatal outcomes in twin pregnancies is limited. A population-based study from Ontario, Canada, reported results similar to our study with regard to SMM and also regarding rates of perinatal mortality: rates of rates of preeclampsia, gestational diabetes and preterm birth at $<32$ weeks were higher in obese women compared with women of normal BMI. ${ }^{11}$ Increased risk of GDM has been consistently reported in women with elevated BMI and twin pregnancy. ${ }^{12,24-27}$ Some studies have also shown an increased risk of pre-eclampsia in obese women ${ }^{24-26}$ however, these findings have not been consistent ${ }^{28}$. Our results suggest that although obese women with twins may have higher rates of gestational hypertension and GDM than those with normal BMI, this does not lead to higher rates of severe pre-eclampsia, eclampsia, and SMM.

With regards to perinatal outcomes, a large US-based study found approximately $50 \%$ higher rates of stillbirth in obese (vs. normal BMI) women with twins, ${ }^{29}$ while others found no association between prepregnancy BMI and a broader composite of adverse perinatal outcomes, including stillbirth and neonatal death. ${ }^{11}$ Our study confirms the absence of elevated risks of adverse perinatal outcomes among obese women, and this contrasts with the consistently strong association between obesity and adverse perinatal outcomes in singleton pregnancies. ${ }^{30-33}$

Clinical and research implications

The absence of an increase in adverse birth outcomes in women with elevated BMI and twin pregnancy is somewhat unexpected given the strong nature of this relationship among singleton pregnancies. Several possible explanations could underlie our findings. Firstly, obese women are at higher risk of infertility, and infertility treatments typically results in dichorionic twins which have fewer adverse pregnancy outcomes compared with monochorionic twins. ${ }^{3435,36}$ However, we observed no difference in use of ART in women with higher BMI, and our sensitivity analysis restricted to opposite sex twins (which are only di-chorionic) showed similar result to our primary analysis. Secondly, obese women have larger fetuses on average and lower rates of small-for-gestational-age (SGA) infants, which can lead to a reduction in SGA-related adverse perinatal outcomes. This effect of obesity may be more evident in twins which have higher baseline rates of SGA. Thirdly, it is possible that maternity care providers may perceive obese women with twins to be at high risk due to two major risk factors and opt for heightened fetal surveillance and intervention. Finally, a selection bias could potentially contribute to the unexpected findings. Our cohort included only those women who continued to be pregnant at 20 weeks' gestation, and women who miscarried were not included in our study. If overweight and obese women with twin pregnancies who had higher propensity for adverse perinatal outcomes had also higher rates of miscarriage, it is possible that twin fetuses with higher survival potential reached 20 weeks' gestation and were included in our study. This would lead to a survival bias and a similar hypothesis has been suggested for other puzzling findings in perinatal epidemiology (such as the lower rate of pre-eclampsia among women who smoke). ${ }^{37,38}$ Additionally, as with any observational cohort, unmeasured confounders (such as nutrition, race/ethnicity and socioeconomic status) may also lead 
to spurious associations or the lack thereof. ${ }^{39}$ However, such factors would have to have differential effects in twin vs singleton pregnancies to explain our findings. All of these possible explanations may also play a role in other similarly paradoxical associations, for instance, a lack of association between advanced maternal age and adverse perinatal outcomes in twins: while advanced maternal age is a strong risk factor for perinatal death in singletons, such an association has not been observed in twins. ${ }^{40,41}$ On the other hand, young maternal age has been consistently associated with the highest maternal age-specific rates of adverse birth outcomes in twins. ${ }^{40,41}$

The most concerning finding in our study relates to the high rates of neonatal death $(2.7 \%)$ and serious neonatal morbidity $(5.5 \%)$ in underweight women with twin pregnancies. These findings are consistent with those from previous studies ${ }^{11,42}$ and possible causes of both low BMI and adverse outcomes include poor nutrition (itself associated with anemia, preterm delivery and low birth weight ${ }^{43} 44,45$ ), anorexia nervosa and substance use. ${ }^{46}$ We did not have specific information about maternal nutrition, however, we observed higher rates of anemia and smoking and alcohol/drug use in underweight women vs. those of other BMI categories. Future studies are needed to validate these findings and explore causal pathways between low BMI and adverse birth outcomes, and to guide targeted interventions.

Strengths and limitations

Our study has several strengths including the detailed population level data on both pregnancy characteristics and outcomes. There is limited evidence in literature regarding the frequency of many of the maternal and neonatal morbidity types among twin pregnancies, especially in relation to pre-pregnancy BMI. We applied newer modeling techniques to flexibly model the relationship between BMI and adverse outcomes, and provided two opportunities (i.e., exposure and outcome models) to optimize adjustment for confounding.

Our study also has several limitations. First, information about pre-pregnancy BMI was missing in 30\% of women. Sensitivity analyses, including multiple imputation, did not alter the results substantially. The only exceptions were in underweight women, where the TMLE-adjusted risks of SMM and stillbirth were higher that the unadjusted risks, and the adjusted risks from both the regression analyses and the imputation analyses. This is likely due to the small number of outcomes in this group. This situation can lead to a trade-off between the improvement from the flexibility of TMLE with the cost of increased volatility in estimates. In such cases, triangulation by other methods (e.g., regression model, as done in our study) is recommended and results need to be interpreted cautiously. ${ }^{47}$ Second, we were unable to completely stratify the study population by chorionicity, which could have confounded or modified the effects of BMI on adverse perinatal outcomes. Third, height and weight information was obtained routinely during prepregnancy and early pregnancy physician visits, and this information was self-reported. Behavioural factors including smoking, alcohol/substance use was also self-reported, and thus subject to a recall bias. Such nondifferential misclassification between weight classes could have biased the results towards null (i.e., leading to an underestimation of the aRR). Fourth, due to a relatively smaller number of women with high BMI, we were unable to stratify obese women into class I, II and III. However, when we examined BMI continuously, we did not find increased risk in those with very high BMI $\left(>35 \mathrm{~kg} / \mathrm{m}^{2}\right)$, although confidence intervals were wide at the BMI range. We were also unable to examine associations with maternal death and specific SMMs, as these were very rare. Finally, as with any observational study, unmeasured confounders (e.g., nutritional status and race/ethnicity) may in part explain some of our results.

\section{Conclusion}

In this population-based cohort study, overweight and obese women did not have an increased risk of SMM or adverse perinatal outcome, compared with women with twin pregnancies who had normal BMI. Underweight women had elevated risks of preterm birth, neonatal death and neonatal morbidity.

The association between pre-pregnancy BMI and adverse perinatal outcomes thus appears to be different in twin compared with singleton pregnancies. These findings have implications for pre-pregnancy counselling and pregnancy care, especially among underweight women with twin pregnancy. Future research is needed to elucidate reasons behind the unexpected differences in adverse perinatal outcomes between singleton and 
twin pregnancies in women with high pre-pregnancy BMI.

\section{Contribution to Authorship}

SL and JB designed the study and formulated the research question. JB analyzed the data and wrote the first draft of the manuscript with supervision from SL. KS, LAM, LW and CM read and reviewed the manuscript and provided critical feedback and suggested additional analyses. All authors approved the final version.

\section{Details of ethics approval}

Ethics approval for this study was obtained from the University of British Columbia - Children's and Women's Hospital and Health Centre of British Columbia Research Ethics Board (H18-03154).

\section{Conflict of interest:}

The authors report no conflict of interests.

\section{Funding :}

This study was supported by funding from the Sick Kids Foundations (grant number SKF-154852). JB is supported by a four-year PhD fellowship award from the University of British Columbia (award number 6456). KSJ is supported by an Investigator award from the BC Children's Hospital Research Institute. SL is supported by a scholar award from the Michael Smith Foundation for Health Research.

The funders had no role in study design, data collection and analysis, decision to publish, or preparation of the manuscript. All inferences, opinions and conclusions drawn in this paper are those of the authors and do not reflect the opinions or policies of the Data Stewards.

\section{References:}

1 Chen C, Xu X, Yan Y. Estimated global overweight and obesity burden in pregnant women based on panel data model. PLoS ONE 2018;13 . DOI:10.1371/journal.pone.0202183.

2 Driscoll AK. Increases in Prepregnancy Obesity: United States, 2016-2019. 2020; : 8.

3 Devlieger R, Benhalima K, Damm P, et al. Maternal obesity in Europe: where do we stand and how to move forward?: A scientific paper commissioned by the European Board and College of Obstetrics and Gynaecology (EBCOG). Eur J Obstet Gynecol Reprod Biol 2016;201 : 203-8.

4 Heslehurst N, Rankin J, Wilkinson JR, Summerbell CD. A nationally representative study of maternal obesity in England, UK: trends in incidence and demographic inequalities in 619323 births, 1989-2007.Int $J$ Obes 2010; $34: 420-8$.

5 Lisonkova S, Muraca GM, Potts J, et al. Association Between Prepregnancy Body Mass Index and Severe Maternal Morbidity. JAMA2017; $318:$ 1777-86.

6 Aune D, Saugstad OD, Henriksen T, Tonstad S. Maternal Body Mass Index and the Risk of Fetal Death, Stillbirth, and Infant Death: A Systematic Review and Meta-analysis. JAMA 2014; 311 : 1536-46.

7 Chu SY, Kim SY, Lau J, et al. Maternal obesity and risk of stillbirth: a metaanalysis. Am J Obstet Gynecol 2007;197 : 223-8.

8 Cheong-See F, Schuit E, Arroyo-Manzano D, et al. Prospective risk of stillbirth and neonatal complications in twin pregnancies: systematic review and meta-analysis. BMJ 2016; 354 : i4353.

9 Madar H, Goffinet F, Seco A, Rozenberg P, Dupont C, Deneux-Tharaux C. Severe Acute Maternal Morbidity in Twin Compared With Singleton Pregnancies. Obstet Gynecol 2019; 133 : 1141-50.

10 Chauhan SP, Scardo JA, Hayes E, Abuhamad AZ, Berghella V. Twins: prevalence, problems, and preterm births. Am J Obstet Gynecol2010; 203 : 305-15. 
11 Ram M, Berger H, Lipworth $\mathrm{H}$, et al. The relationship between maternal body mass index and pregnancy outcomes in twin compared with singleton pregnancies. Int J Obes 2020; $44: 33-44$.

12 Al-Obaidly S, Parrish J, Murphy KE, Maxwell C. Maternal pre-gravid body mass index and obstetric outcomes in twin gestations. $J$ Perinatol 2014; $34: 425-8$.

13 BC Perinatal Data Registry (BCPDR) | www.popdata.bc.ca. https://www.popdata.bc.ca/data/health/PSBC (accessed June 11, 2020).

14 Frosst G, Hutcheon J, Joseph K, Kinniburgh B, Johnson C, Lee L. Validating the British Columbia Perinatal Data Registry: a chart re-abstraction study. BMC Pregnancy Childbirth 2015; 15 . DOI:10.1186/s12884015-0563-7.

15 Dzakpasu S, Deb-Rinker P, Arbour L, et al. Severe maternal morbidity surveillance: Monitoring pregnant women at high risk for prolonged hospitalisation and death. Paediatr Perinat Epidemiol2020; 34 : 427-39.

16 VanderWeele TJ. Principles of confounder selection. Eur J Epidemiol 2019; 34 : 211-9.

17 Greenland S, Pearce N. Statistical Foundations for Model-Based Adjustments. Annu Rev Public Health 2015; 36 : 89-108.

18 Targeted Learning - Causal Inference for Observational and Experimental Data | Mark J. van der Laan | Springer. https://www.springer.com/gp/book/9781441997814 (accessed April 12, 2021).

19 Balzer LB, Zheng W, van der Laan MJ, Petersen ML. A new approach to hierarchical data analysis: Targeted maximum likelihood estimation for the causal effect of a cluster-level exposure. Stat Methods Med Res 2019; 28 : 1761-80.

20 Zou GY, Donner A. Extension of the modified Poisson regression model to prospective studies with correlated binary data. Stat Methods Med Res 2013; 22 : 661-70.

21 Gill P, Lende MN, Van Hook JW. Twin Births. In: StatPearls. Treasure Island (FL): StatPearls Publishing, 2021. http://www.ncbi.nlm.nih.gov/books/NBK493200/ (accessed Oct 8, 2021).

22 R Core Team (2020). R: A language and environment for statistical computing. R Foundation for Statistical Computing. Vienna, Austria. URL https://www.R-project.org/.

23 Benkeser D, Carone M, Laan MJVD, Gilbert PB. Doubly robust nonparametric inference on the average treatment effect.Biometrika 2017; $104: 863-80$.

24 Fox NS, Roman AS, Saltzman DH, Klauser CK, Rebarber A. Obesity and adverse pregnancy outcomes in twin pregnancies. J Matern-Fetal Neonatal Med Off J Eur Assoc Perinat Med Fed Asia Ocean Perinat Soc Int Soc Perinat Obstet 2014; 27 : 355-9.

25 Lucovnik M, Blickstein I, Verdenik I, Trojner-Bregar A, Tul N. Maternal obesity in singleton versus twin gestations: a population-based matched case-control study. J Matern Fetal Neonatal Med 2015;28 : 623-5.

26 Lučovnik M, Tul N, Verdenik I, Novak Z, Blickstein I. Risk factors for preeclampsia in twin pregnancies: a population-based matched case-control study. J Perinat Med 2012; 40 : 379-82.

27 Simões T, Queirós A, Correia L, Rocha T, Dias E, Blickstein I. Gestational diabetes mellitus complicating twin pregnancies. 2011;39: 437-40.

28 Suzuki S, Yoneyama Y, Sawa R, Shin S, Araki T. Clinical Usefulness of Maternal Body Mass Index in Twin Pregnancies. Hypertens Pregnancy2000; 19 : 273-9.

29 Salihu HM, Alio AP, Belogolovkin V, et al. Prepregnancy Obesity and Risk of Stillbirth in Viable Twin Gestations. Obesity2010; $18:$ 1795-800.

30 Yao R, Ananth CV, Park BY, Pereira L, Plante LA. Obesity and the risk of stillbirth: a population-based cohort study. Am J Obstet Gynecol 2014; 210 : 457.e1-457.e9. 
31 Lu GC, Rouse DJ, DuBard M, Cliver S, Kimberlin D, Hauth JC. The effect of the increasing prevalence of maternal obesity on perinatal morbidity. Am J Obstet Gynecol 2001; 185 : 845-9.

32 Scott G, Gillon TE, Pels A, Dadelszen P von, Magee LA. Guidelines-similarities and dissimilarities: a systematic review of international clinical practice guidelines for pregnancy hypertension.Am J Obstet Gynecol 2020; 0 . DOI:10.1016/j.ajog.2020.08.018.

33 Kristensen J, Vestergaard M, Wisborg K, Kesmodel U, Secher NJ. Pre-pregnancy weight and the risk of stillbirth and neonatal death.BJOG Int J Obstet Gynaecol 2005; $112: 403-8$.

34 Marleen S, Dias C, Nandasena R, et al. Association between chorionicity and preterm birth in twin pregnancies: a systematic review involving 29864 twin pregnancies. BJOG Int J Obstet Gynaecol2021; 128 : 788-96.

35 Salihu HM, Dunlop A-L, Hedayatzadeh M, Alio AP, Kirby RS, Alexander GR. Extreme Obesity and Risk of Stillbirth Among Black and White Gravidas. Obstet Gynecol 2007; 110 : 552-7.

36 Pasquali R, Patton L, Gambineri A. Obesity and infertility.Curr Opin Endocrinol Diabetes Obes 2007; $14: 482-7$.

37 Lisonkova S, Joseph KS. Left Truncation Bias as a Potential Explanation for the Protective Effect of Smoking on Preeclampsia.Epidemiol Camb Mass 2015; 26 : 436-40.

38 Sapra KJ, Chaurasia AK, Hutcheon JA, Ahrens KA. Reconstructing a Pregnancy Cohort to Examine Potential Selection Bias in Studies on Racial Disparities in Preterm Delivery. Paediatr Perinat Epidemiol 2017; $31: 55-63$.

39 Hernan MA, Robins J. Causal Inference: What if. Boca Raton: Chapman \& Hill/CRC. 2020.

40 Lisonkova S, Joseph KS, Bell R, Glinianaia SV. Effect of advanced maternal age on perinatal outcomes in twins: the impact of chorionicity. Ann Epidemiol 2013; 23 : 428-34.

41 Lisonkova S, Sheps SB, Janssen PA, Lee SK, Dahlgren L. Effect of older maternal age on birth outcomes in twin pregnancies: a population-based study. J Perinatol Off $J$ Calif Perinat Assoc2011; 31 : 85-91.

42 Sung SJ, Lee SM, Kim S, et al. The Risk of Spontaneous Preterm Birth according to Maternal Pre-pregnancy Body Mass Index in Twin Gestations. J Korean Med Sci 2018; 33 . DOI:10.3346/jkms.2018.33.e103.

43 Gete DG, Waller M, Mishra GD. Effects of maternal diets on preterm birth and low birth weight: a systematic review. Br J Nutr 2020;123 : 446-61.

44 Smith C, Teng F, Branch E, Chu S, Joseph KS. Maternal and Perinatal Morbidity and Mortality Associated With Anemia in Pregnancy.Obstet Gynecol 2019; 134 : 1234-44.

45 Jung J, Rahman MM, Rahman MS, et al. Effects of hemoglobin levels during pregnancy on adverse maternal and infant outcomes: a systematic review and meta-analysis. Ann N Y Acad Sci 2019;1450 : 69-82.

46 Ante Z, Luu TM, Healy-Profitós J, et al. Pregnancy outcomes in women with anorexia nervosa. Int $J$ Eat Disord 2020; $53:$ 403-12.

$47 \mathrm{Yu}$ Y-H, Bodnar LM, Brooks MM, Himes KP, Naimi AI. Comparison of Parametric and Nonparametric Estimators for the Association Between Incident Prepregnancy Obesity and Stillbirth in a Population-Based Cohort Study. Am J Epidemiol 2019; 188 : 1328-36. 
figures/Figure1/Figure1-eps-converted-to.pdf 